\title{
Wen
}

\section{LORENTZ-INVARIANT FORMULATION OF CHERENKOV RADIATION BY TACHYONS}

(NASA-TM-X-65851) LORENTZ-INVARIANT
FORMDLATION OF CHERENKOV RADIATION

TACHYONS F.C. JONES (NASA)

$34 \mathrm{p}$. Jones (NASA) Mar. 1972

\author{
N72-19766 \\ G3/24 Unclas 21042
}

CSCL $20 \mathrm{H}$

\section{FRANK C. JONES}
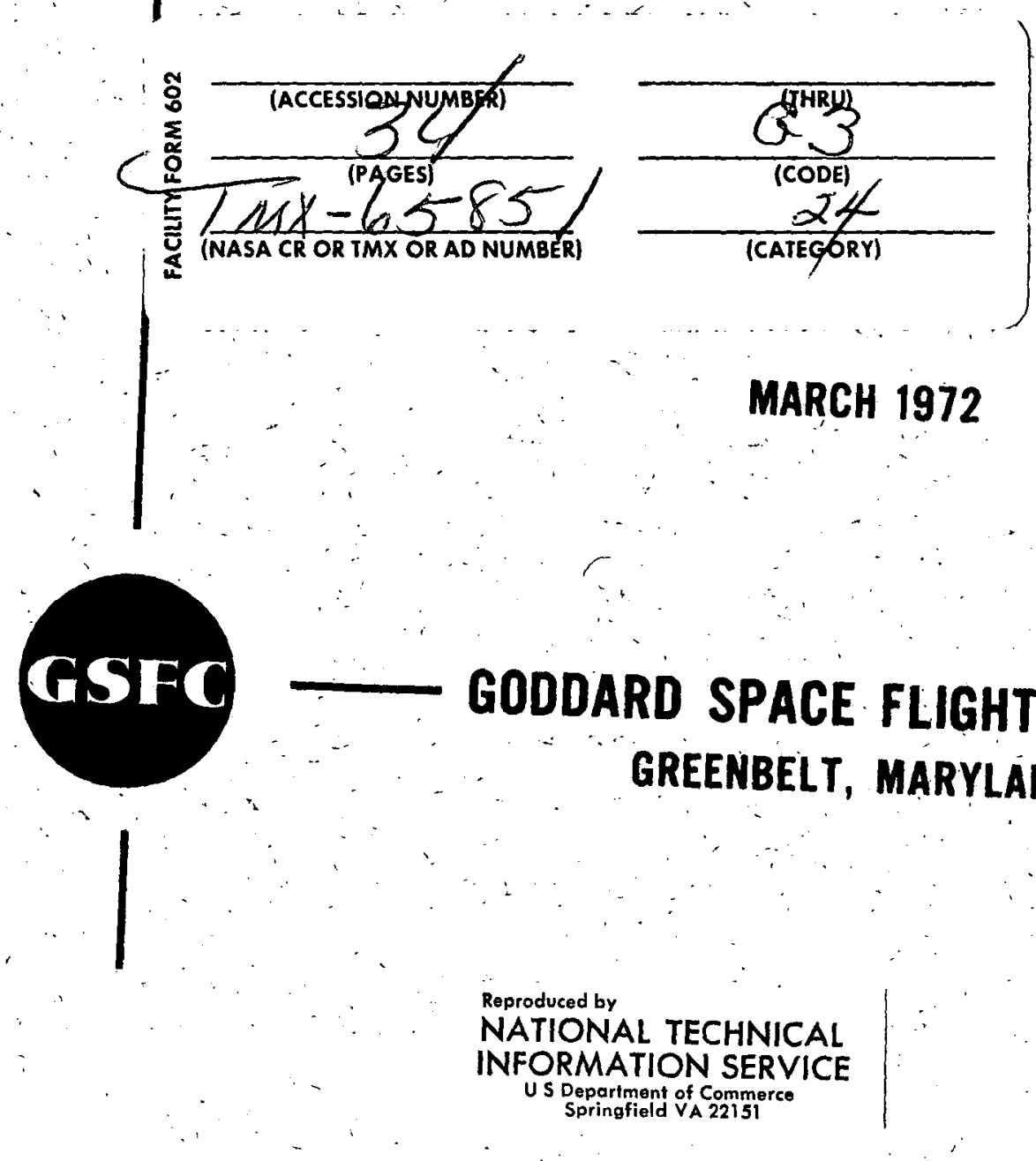

MARCH 1972

NATIONAL TECHNICAL 


\title{
LORENTZ-INVARIANT FORMULATION OF CHERENKOV RADIATION BY TACHYONS
}

\author{
Frank C. Jones
}

Theoretical Studies Branch

March 1972

NASA Goddard Space Flight Center

Greenbelt, Maryland 20771 


\title{
a LORENTZ-INVARIANT FORMULATION OF \\ CHERENKOV RADIATION BY TACHYONS
}

\author{
Frank C. Jones \\ Theoretical Studies Branch \\ NASA Goddard Space Flight Center \\ Greenbelt, Maryland 20771
}

\begin{abstract}
Previous treatments of Cherenkov radiation, electromagnetic and gravitation, by tachyons have been in error because the prescription employed to cut off the divergent integral over frequency, namely $\omega_{\max }=\mathrm{E} / \mathrm{\hbar}$, is not a Lorentz invariant procedure. The resulting equation of motion for the tachyon is therefore not covariant. The proper procedure requires an extended, deformable distribution of charge or mass and yields a particularly simple form for the tachyon's world line, one that could have been deduced from simple invariance considerations. It is shown that Cherenkov radiation by tachyons implys their ultimate annihilation with an antitachyon and demonstrates a disturbing property of tachyons, namely the impossibility of specifying arbitrary Cauchy data even in a purely classical theory.
\end{abstract}


CONTENTS

Page

ABSTRACT $\ldots \ldots \ldots \ldots \ldots \ldots \ldots \ldots \ldots \ldots \ldots \ldots \ldots \ldots \ldots$

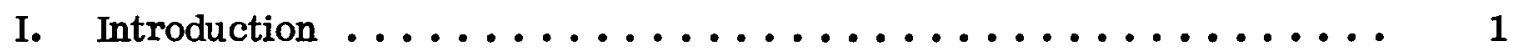

II. Lorentz Invariant Form of the Drag Force . . . . . . . . . 3

III. Derivation From Invariance $\ldots \ldots \ldots \ldots \ldots \ldots \ldots \ldots$

IV. The Effect of Acceleration................. 12

v. Discussion ........................ 14

Acknowledgments.......................... 19

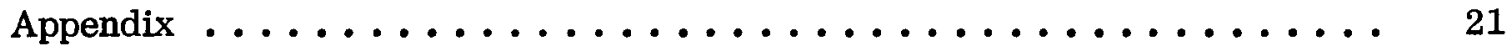

REFERENCES....................... 27 


\section{LORENTZ-INVARIANT FORMULATION OF CHERENKOV RADIATION BY TACHYONS}

\section{Introduction}

Since their introduction ${ }^{1-3}$ into the literature of physics a few years ago, tachyons have stirred a lively debate among physicists. The debate has centered about the question of whether or not the existence of particles that travel with a velocity $v>1$ (we employ units in which $c=1$ ) would produce certain paradoxes concerning the concept of causality. In a field theory of tachyons ${ }^{1,3,4}$ field commutators do not vanish for space-like separations. This makes it impossible to specify arbitrary Cauchy data for the field and has raised questions ${ }^{3,5}$ concerning the localizability of tachyons. Problems concerning unitarity have also been raised.

Certain authors ${ }^{7}$ have raised the question of whether the field equations that have been proposed would indeed exhibit superluminal effects and have suggested that the correct interpretation should be in terms of unstable modes. It appears ${ }^{8}$ however that this issue is one of choosing those boundary conditions that make the solutions describe the sort of phenomena that one wishes to describe. It would appear that if a consistent, and hence paradox free, classical theory of tachyons exists, then an equivalent quantum field theory should exist also (at least to the extent that they exist for ordinary particles). We shall therefore consider only a classical (unquantized) picture of tachyons in this paper. 
The primary objection that has been raised against the existence of classical tachyons is the possibility that using these particles one could propagate information backwards in time thereby creating causal loops 9,10 as paradoxes. The sort of images that are conjured up by this possibility is illustrated by the fact that in one recent discussion ${ }^{11}$ in the literature almost half of the references cited were to science fiction stories. All of the previous discussions of this question have employed situations in which tachyons were absorbed and reemitted (or scattered) by at least two observers in order to produce the causal loop.

We shall not discuss such causal loops in this paper, rather we shall show that if we consider the Cherenkov radiation emitted by tachyons a further example of such questions concerning causality arise in the emission of even a single tachyon. The Cherenkov emission of electromagnetic radiation by charged tachyons has been examined by Alväger and Kreisler ${ }^{12}$ and their results have been applied $^{13}$ in an attempt to detect such particles experimentally. The case of emission of gravitational radiation has been considered by Lapedes and e'acobs ${ }^{14}$ and these authors have applied their results to the events recorded by Weber ${ }^{15}$ and to the question of survival of tachyons from the "big bang" creation of the universe.

Unfortunately these authors have all made the unwarranted assumption that a tachyon cannot radiate a photon or graviton that has more energy than the tachyon itself possesses. While this requirement is quite sensible for radiation 
by normal particles, the existence of negative energy states for tachyons ${ }^{2,4}$ makes such a requirement unjustifiable in this case. In fact, this assumption leads to an equation of motion for the tachyon that is not Lorentz invariant ${ }^{16}$ and would hence single out a preferred reference frame if it were correct. In section II we shall derive a properly Lorentz covariant form for the Cherenkov drag force on a charged tachyon. In section III we shall show how the form of the acceleration, and hence the drag force, can be obtained, up to a multiplicative constant, from considerations of Lorentz invariance alone. In Section IV the effect of acceleration on the drag force will be discussed (we conclude that it has no effect) and in Section $\mathrm{V}$ we will discuss the resulting form of the world line of a "free" tachyon and estimate the effect of Cherenkov emission of gravitational waves by a neutral tachyon. We will see that this world line implies that a tachyon, if left to itself, must always annihilate with an anti-tachyon and as a consequence arbitrary Cauchy data may not be specified for a "free" tachyon even in the classical case.

\section{Lorentz Invariant Form of the Drag Force}

It was first pointed out by Sommerfeld ${ }^{17}$ that a charged particle moving with a uniform velocity $v>1$ would experience a drag force associated with the emission of electromagnetic radiation. With the advent of relativity theory Sommerfelds result was forgotten until Frank and Tamm ${ }^{18,19}$ showed that the phenomenon of Cherenkov radiation was essentially that investigated by Sommerfeld. Indeed, the two theories are mathematically almost identical. ${ }^{20}$ 
In the theory of the Cherenkov effect a particle whose velocity exceeds the speed of light in the medium through which it is passing, $n v>1$ where $n$ is the index of refraction, loses energy according to the formula ${ }^{18,19}$

$$
\frac{d w}{d t}=-v e^{2} \int\left(1-\frac{1}{v^{2} n^{2}(\omega)}\right) \omega d \omega
$$

where $\mathbf{n}(\omega)$ is the frequency dependent index of refraction and the integral extends over all frequencies $(\omega>0)$ for which $\operatorname{vn}(\omega)>1$. In the case of a charged tachyon $\mathrm{v}>1$ and $\mathrm{n}=1$ independent of frequency we therefore have

$$
\frac{d w}{d t}=\frac{-e^{2}\left(v^{2}-1\right)}{v} \int_{0}^{\infty} \omega d \omega
$$

which is clearly divergent. This is related to the fact, well known to Sommerfeld ${ }^{17}$ that the electromagnetic field of a point charge is singular on the shock front or "Mach cone" having the particle as its apex. This leads to a divergent expression for the Poynting vector of the radiation and hence for the retarding or drag force on the particle.

Until now the solution to this problem employed in the literature ${ }^{12,14}$ has been to appeal to the quantum nature of the emission process and the statement that a particle cannot emit a photon with more energy than the particle possesses. With this principle we have 


$$
\begin{aligned}
\frac{d w}{d t} & =\frac{-e^{2}\left(v^{2}-1\right)}{v} \int_{0}^{E / h} \omega d \omega \\
& =\frac{-e^{2}\left(v^{2}-1\right) E^{2}}{2 v \hbar^{2}}=-v\left(\frac{e^{2} \mu^{2}}{2 \hbar^{2}}\right) \frac{E^{2}}{p^{2}}
\end{aligned}
$$

where $\mu$ is the "rest" mass of the tachyon and $\mathrm{p}$ is its momentum

$$
p=\frac{\mu v}{\left(v^{2}-1\right)^{1 / 2}}
$$

However, this result is clearly not Lorentz invariant ${ }^{16}$; a particle losing energy as in (3) would asymptotically approach zero energy. Zero energy is not an invariant notion, however; in another Lorentz frame the particle would be seen to approach some other, non-zero, energy in violation of (3). Hence equation (3) cannot be a law of nature describing a particle moving in free space.

On closer inspection the principle on which (3) was based can be seen to be incorrect. Since negative energy states of tachyons can be obtained from positive energy states by a Lorentz transformation ${ }^{2,4}$ the requirement $\hbar \omega \leq \mathrm{E}$ cannot be an invariant one. For if it is fulfilled in one Lorentz frame, one can always find a frame in which the recoil tachyon has negative energy and hence the condition is violated. According to the reinterpretation principle ${ }^{2,4}$ the process would appear as a tachyon-antitachyon annihilation process in the latter frame.

One might be led to believe that the condition $\hbar \omega \leq \mathrm{E}$ could be employed by ruling out annihilation processes from consideration, but for tachyons such a 
separation cannot be made in an invariant manner and any properly Lorentz invariant treatment of one process must automatically include the other.

The essential solution to the problem was also known to Sommerfeld. He pointed out that one could obtain a finite drag force if one considered an extended charge distribution. He obtained a drag force energy loss formula

$$
\frac{d w}{d t}=-\frac{9 e^{2}\left(v^{2}-1\right)}{4 a_{0} v}
$$

which is also not Lorentz invariant.

The reason for the non-invariance of (4) is simply that Sommerfeld considered a rigid spherical distribution of charge with radius $a_{0}$ which is a prerelativity concept. Clearly what one must do to obtain an invariant expression for $\mathrm{dw} / \mathrm{dt}$ is to consider not a rigid sphere but a deformable charge distribution whose shape undergoes a Lorentz extension (it is an extension for particles with $\mathrm{v}>1$ rather than a contraction as in the usual case) ${ }^{2}$. Such a distribution would be given by

$$
\rho(v, \mathbf{x}, \mathbf{y}, \mathbf{z})=\rho\left(\mathbf{x}, \mathbf{y}, \gamma_{\mathbf{s}} \mathbf{z}\right)
$$

where $\gamma_{\mathrm{s}} \equiv\left(\mathrm{v}^{2}-1\right)^{-1 / 2}$ and $\mathrm{v}$ is in the $\mathrm{z}$ direction.

In the appendix we show that the effect of a (cylindrically symmetric) distributed charge on equation (2) is to replace it with

$$
\frac{\mathrm{d} w}{\mathrm{dt}}=-\mathrm{v}\left(\mathrm{v}^{2}-1\right) \int_{0}^{\infty}\left|\tilde{\rho}\left(\frac{\mathrm{k}_{z}}{\gamma_{\mathrm{s}}}, \mathrm{k}_{\mathrm{z}}\right)\right|^{2} \mathrm{k}_{\mathrm{z}} \mathrm{dk} \mathrm{z}
$$


where $\tilde{\rho}\left(\mathrm{k}_{\perp}, \mathrm{k}_{\mathbf{z}}\right)$ is the Fourier-Bessel transform of the charge distribution, i.e.

$$
\rho(r, z)=\frac{1}{4 \pi^{2}} \int_{-\infty}^{\infty} d k_{z} \int_{0}^{\infty} k_{\perp} d k_{\perp} \exp \left(i k_{z} z\right) J_{0}\left(k_{\perp} r\right) \tilde{\rho}\left(k_{1}, k_{z}\right)
$$

and $\omega=v k_{z}$. For a point charge $\tilde{\rho}\left(k_{\perp}, k_{z}\right)=e$ and we recover (2).

We shall now assume with Sommerfeld that

$$
\widetilde{\rho}\left(\mathrm{k}_{1}, \mathrm{k}_{\mathrm{z}}\right)=\widetilde{\rho}(\mathrm{k})=\frac{3 \mathrm{e}}{\left(\mathrm{k} \mathrm{a}_{0}\right)^{3}}\left[\sin \mathrm{k} \mathrm{a}_{0}-\left(\mathrm{k} \mathrm{a}_{0}\right) \cos \mathrm{k} \mathrm{a}_{0}\right]
$$

where $k=\left(k_{\perp}^{2}+k_{z}^{2}\right)^{1 / 2}$ for a rigid sphere of radius $a_{0}$ and $k=\left(k_{\perp}^{2}+k_{z}^{2} / \gamma_{s}^{2}\right)^{1 / 2}$ for a Lorentz deformable sphere. For the quantity in equation (6) we have

$$
\tilde{\rho}=\left(\frac{k_{z}}{\gamma_{s}}, k_{z}\right)=\tilde{\rho}\left(k^{\prime}\right)
$$

where

$$
\mathrm{k}^{\prime}=\left[\left(\mathrm{v}^{2}-1\right) \mathrm{k}_{\mathrm{k}}^{2}+\mathrm{k}_{\mathrm{z}}^{2}\right]^{1 / 2}=\mathrm{vk}_{\mathrm{z}}
$$

for a rigid sphere and

$$
\mathrm{k}^{\prime}=\left[\left(\mathrm{v}^{2}-1\right) \mathrm{k}_{\mathrm{z}}^{2}+\left(\mathrm{v}^{2}-1\right) \mathrm{k}_{\mathrm{z}}^{2}\right]^{1 / 2}=\sqrt{2} \mathrm{k}_{\mathrm{z}} / \gamma_{\mathrm{s}}
$$

for a deformable one. Inserting expression (8) for $\tilde{\rho}$ in equation (6) yields upon integration 


$$
\frac{d w}{d t}=-\frac{9 e^{2}}{4 a_{0}^{2}} \frac{\left(v^{2}-1\right)}{v} \text { for a rigid sphere }
$$

and

$$
\frac{d w}{d t}=-\frac{9 e^{2}}{8 a_{0}^{2}} v \text { for a deformable sphere }
$$

We shall see that equation (9) is a Lorentz invariant expression that leads to an invariant world line for a charged tachyon moving in a vacuum.

To see that (9) is invariant we note that $\gamma_{s} d / d t=d / d \tau$ where $\tau$ is the proper length (not time) of the tachyons world line and that w is the fourth component of the 4-momentum $P=(\vec{p}, w)$. We may therefore write

where

$$
\frac{d P}{d \tau}=-\left(\frac{9 e^{2}}{8 a_{0}^{2}}\right) \frac{(\hat{v}, v)}{\left(v^{2}-1\right)^{1 / 2}}=\mu A
$$

$$
A=-\frac{1}{\mu}\left(\frac{9 \mathrm{e}^{2}}{8 \mathrm{a}_{0}^{2}}\right) \frac{(\hat{\mathrm{v}}, \mathrm{v})}{\left(\mathrm{v}^{2}-1\right)^{1 / 2}}
$$

is the 4-acceleration of the particle. Equation (10) is a 4-vector equation and hence covariant.

III. Derivation From Invariance

The form of equation (10) can be inferred simply from considerations of Lorentz invariance combined with the fact that in empty space the only direction that can be unambiguously defined is that of the particle's velocity. Consider a free charged tachyon in empty space, its acceleration can depend on nothing but 
its velocity with perhaps an overall constant depending upon intrinsic properties of the particle. Moreover, it must depend on the particle's 4-velocity in a Lorentz covariant manner. This means that the dependence must be of the form

$$
A=A(U)
$$

where $A$ is the 4-acceleration and $U$ is the 4-velocity. Now since two 4-velocities $U^{\prime}$ and $U$ are related by a Lorentz transformation $U^{\prime}=\Gamma U$ we must have, since $A$ is a 4-vector

$$
A\left(U^{\prime}\right)=A(\Gamma U)=\Gamma A\left(U^{\prime}\right)=A^{\prime}
$$

In other words, to go from $A(U)$ to $A\left(U^{\prime}\right)$ one simply applies the Lorentz transformation to A that carried $U$ into $U^{\prime}$. Since the length of a 4-vector is preserved under a Lorentz transformation we have

$$
A^{2}\left(U^{\prime}\right)=A^{2}(U)=\text { cons } t .
$$

Since the 4-acceleration is always orthogonal to the 4-velocity we also have

$$
A \cdot U=0 .
$$

Since A must be a unique function of $U$ we may write

$$
A=f(\vec{v})\left(\hat{a}, a_{4}\right)
$$


where $f(\vec{v})$ is an as yet unspecified function of the three velocity and $a$ is a unit three vector. From (13) we have $a_{4}=\hat{a} \cdot \vec{v}$ and since â must be uniquely specified by $\vec{v}$ we must have $\hat{a}=\hat{v}$. From (12) we have

$$
f^{2}(\vec{v})\left(1-v^{2}\right)=- \text { cons } t .
$$

so we are led to the final form

$$
A=\operatorname{cons} t . \times \frac{(\hat{v}, v)}{\left(v^{2}-1\right)^{1 / 2}}
$$

which is of the same form as equation (10).

Since

$$
\mathrm{A} \equiv \frac{\mathrm{d} U}{\mathrm{~d} \tau}=\gamma_{\mathrm{s}} \frac{\mathrm{d}}{\mathrm{d} t} \gamma_{\mathrm{s}}(\overrightarrow{\mathrm{v}}, 1)
$$

we have

$$
A=-\left(\frac{d \vec{v}}{d t}, \vec{v} \cdot \frac{d \vec{v}}{d t}\right) /\left(v^{2}-1\right)^{2}
$$

so

$$
-\frac{d \vec{v}}{d t} /\left(v^{2}-1\right)^{2}=-k \hat{v} /\left(v^{2}-1\right)^{1 / 2}
$$

and

$$
\frac{d \vec{v}}{d t}=k \hat{v}\left(v^{2}-1\right)^{3 / 2}
$$

This may be integrated to give

$$
\frac{\vec{v}}{\left(v^{2}-1\right)^{1 / 2}}=-k\left(t-t_{0}\right) \hat{v}
$$


which may be solved to give

$$
v \equiv \frac{d z}{d t}= \pm \frac{k\left(t-t_{0}\right)}{\left[k^{2}\left(t-t_{0}\right)^{2}-1\right]^{1 / 2}}
$$

Substituting $k\left(t-t_{0}\right)=s$ we have

$$
\mathrm{k} \frac{\mathrm{d} z}{\mathrm{ds}}=\frac{ \pm \mathrm{s}}{\left(\mathrm{s}^{2}-1\right)^{1 / 2}}
$$

which may be integrated once again to give

$$
k\left(z-z_{0}\right)= \pm\left(s^{2}-1\right)^{1 / 2}
$$

or

$$
k^{2}\left(z-z_{0}\right)^{2}=s^{2}-1=k^{2}\left(t-t_{0}^{2}\right)-1
$$

or

$$
\left(z-z_{0}\right)^{2}-\left(t-t_{0}\right)^{2}=-1 / k^{2}
$$

and the world line is the invariant hyperbola whose asymptotes are the light cone with apex at $x_{0}, t_{0}$.

This demonstrates that the energy loss formula (9) and the resulting acceleration (10) do yield an invariant equation of motion and world line for a tachyon in a vacuum in contrast to the previously used formula (3). 
IV. The Effect of Acceleration

Our expression for the energy loss rate (9) and resulting acceleration (10) of a charged tachyon in free space have been derived in a standard manner for describing Cherenkov radiation, namely by assuming the particle to be unaccelerated. Such a derivation would appear to be inherently self-contradictory; a finite acceleration is derived by assuming no acceleration. The results of the calculation are not necessarily wrong, however, provided one can show that the acceleration of the particle does not alter the instantaneous drag force of the radiation reaction. This would not have to be true for an acceleration in general but just for the particular hyperbolic motion derived in section III.

Unfortunately, so far as the present author is aware, there is no general theory of radiation reaction for particles with $v>1$. The well known method of Dirac $^{21}$ does not appear to be applicable in this situation. ${ }^{22}$ In this method the radiation field is assumed to be given by

$$
F_{r a d}^{i j}=\frac{1}{2}\left(F_{r e t}^{i j}-F_{a d v}^{i j}\right)
$$

where $F_{r e t}^{i j}$ and $F_{a d v}^{i j}$ are the retarded and advanced solutions of Maxwell's equations. Inserting (21) into the equation of motion

$$
e F^{i j} U_{j}=m A^{i}
$$

yields the well known formula for radiation reaction 


$$
\underset{\text { rad. reac. }}{\gamma^{i}}=\frac{2}{3} e^{2}\left[\frac{d A^{i}}{d \tau}-U^{i}\left(A^{j} A_{j}\right)\right] \text {. }
$$

The existence of the two solutions $F_{r e t}$ and $F_{a d v}$ is due to the fact that a particle with $\mathrm{v}<1$ always intersects the backward light cone of any field point once and the forward light cone of the same field point once. For a particle with a constant $\mathrm{v}>1$, however, the light cones of a given field point may be intersected either twice on the backward cone, twice on the forward cone, or not at all $20,23$.

Therefore such a partition of the field as required by the Dirac method does not seem to be possible.

Teitelboim ${ }^{24}$ has shown that the Lorentz-Dirac equation of motion may be derived without reference to advanced fields. The total field of a particle is singular on the world line of a particle making any straight forward application of (22) meaningless but Teitelboim has shown that for a particle with $\mathrm{v}<1$ this singularity may be separated out (mass renormalization) in an invariant manner by defining the field on the particle's world line using a suitable averaging procedure. Unfortunately, in the case of a point particle with $v>1$ the sing, larity of the field is much worse and the procedure of Teitelboim breaks down. This is due in part to the fact that one of the unit vectors normal to a world line with $\mathrm{v}>1$ is time like and the components of such a vector are unbounded in the averaging procedure.

These difficulties are not surprising considering that the radiation reaction force is infinite for a point particle with $v>1$ even when it is unaccelerated. 
This would indicate that the general solution must be sought within the context of extended particle theories. The obvious complexity of such a theory places it well beyond the scope of the present paper.

However, it is our claim that such a general solution is unnecessary. We have seen that the form of the acceleration, and thus the drag force, can be deduced, up to a multiplicative constant, from very general considerations of invariance. The only thing that must be derived from an explicit theory is this constant coefficient.

From equation (18) we see that the 3 - acceleration becomes arbitrarily small as $\mathrm{v} \rightarrow 1$. In this case the assumption of unaccelerated motion may be made an arbitrarily good one. The energy loss rate (9) however, remains quite finite in this case and may be calculated with arbitrary precision to yield the constant coefficient $\left(9 \mathrm{e}^{2} / 8 \mathrm{a}_{0}^{2}\right)$. This value of the coefficient should be valid, therefore, for any value of $v>1$; (the exact numerical factor $9 / 8$ depends upon the explicit form of the charge distribution and thus should not be taken too seriously).

Because of the above argument we therefore assert that equation (10) is the correct equation of motion for a charged tachyon in free space even though acceleration was neglected in its derivation.

\section{Discussion}

We have seen in the foregoing that for a classical theory of Cherenkov radiation by a charged tachyon to be Lorentz invariant we must consider the 
tachyon to be an extended, deformable particle and that annihilation with an antitachyon must be considered as an intimate part of the same process. Indeed, the only way an observer could interpret the world line of equation (20) under the reinterpretation principle ${ }^{2,4}$ is as representing a particle and antiparticle approaching each other along a common line of motion, each of them losing energy via Cherenkov radiation. At the exact instant that they both become transcendent $(v=\infty, E=0)$ they meet and annihilate at $z=z_{0}, t=t_{0}-1 / K=t_{0}-\left(8 a_{0}^{2} / 9 e^{2}\right)$. There is no annihilation radiation as such since at the moment of annihilation both particles have $\mathrm{E}=0$.

If we consider the distance to the point of inevitable annihilation as the range of the tachyon we may write an extremely simple range-energy formula. Since

$$
\frac{d w}{d z}=\frac{1}{v} \frac{d w}{d t}=-\frac{9 e^{2}}{8 a_{0}^{2}}
$$

the range is given by

$$
R=\frac{8 a_{0}^{2}}{9 e^{2}} E
$$

To obtain any further results we must choose values for the size, charge, etc. of the particle. In the following we shall assume for concreteness that our tachyon has the same charge and mass as the electron and that its size is of the same order as the electron's Compton wavelength. We then have 


$$
\begin{aligned}
\mathrm{R} & \approx\left(\frac{\lambda_{0}^{2} \mu}{\mathrm{e}^{2}}\right)\left(\frac{\mathrm{E}}{\mu}\right)=\left(\frac{\lambda_{0}}{\mathrm{r}_{0}}\right)\left(\frac{\mathrm{E}}{\mu}\right) \lambda_{0} \\
& =137 \lambda_{0}(\mathrm{E} / \mu) \approx 5.5 \times 10^{-9}(\mathrm{E} / \mu) \mathrm{cm} .
\end{aligned}
$$

where $r_{0}=e^{2} / \mu$ the classical electron radius. For $E \approx \mu$ one obtains a range of $5.5 \times 10^{-9} \mathrm{~cm}$ in contrast to the value of $5 \times 10^{-3} \mathrm{~cm}$ obtained by Alväger and Kreisler. ${ }^{12}$ Incidentally, since the energy loss per unit path length is given by the constant $9 e^{2} / 8 a_{0}^{2}$, a tachyon in a constant electric field would not necessarily reach a steady state energy as was claimed by these authors. If on the other hand we give our tachyon the largest energy ever seen in a cosmic-ray particle, $10^{20} \mathrm{eV}$, we obtain a range of $11 \mathrm{~km}$ so we may be sure that such particles do not arrive from astronomical distances.

The above theory applies not only to charged tachyons but to neutral tachyons which should Cherenkov radiate gravitational radiation.14,16,25 The wave equation of general relativity is nonlinear and such nonlinearity would be most manifest in the vicinity of the Cherenkov shock front. However, if the particle is large compared to its Schwarzschild radius, Gm, non-linear effects should be small. We may adapt our range formula (25) to the case of emission of gravitational waves merely by replacing the classical electromagnetic radius $r_{0}$ by the Schwarzschild radius $\mathbf{r}_{\mathrm{s}}$. We obtain 


$$
\begin{aligned}
\mathrm{R} & \approx \frac{\lambda_{0}^{2}}{\mathrm{r}_{\mathrm{s}}} \frac{\mathrm{E}}{\mu}=-2.4 \times 10^{34}(\mathrm{E} / \mu) \mathrm{cm} \\
& =8 \times 10^{15}(\mathrm{E} / \mu) \mathrm{pc} .
\end{aligned}
$$

so such particles could well be of astronomical origin.

If, on the other hand, we consider particles of protonic mass equations (25) and (26) become respectively

$$
\begin{aligned}
R & \approx 3 \times 10^{-12}(\mathrm{E} / \mu) \mathrm{cm} . \\
R & \approx 1.3 \times 10^{6}(\mathrm{E} / \mu) \mathrm{pc} .
\end{aligned}
$$

We can see from the above that charged tachyons will have a range that is quite short even for energies as large as the most energetic cosmic rays. We will discuss the implications of this shortly. Neutral tachyons which are coupled only to the gravitational field have ranges that are of cosmological scale in striking contrast to their charged counterparts. Such particles, however, would be essentially undetectable since to be detectable they must have a coupling to normal matter of a reasonable strength. Such a coupling is characterized by the square of a "change" that is shared by the particle and other matter. Such a "charge" however would mean that the tachyon would emit the appropriate intermediate field via Cherenkov radiation and therefore have a range that was inversely proportional to the square of that charge, i.e. the range against 
emission of mesons via the strong interactions would be $\approx 137$ times shorter than that for photon emission. We must assume, therefore, that any tachyons that we may readily detect or produce in the laboratory will have ranges comparable to or shorter than the electromagnetic ones given by (25) and (25'). This leads to a curious result.

We should first of all note that the total world line of the tachyon must be of finite length. If it is not, it approaches arbitrarily near the light cone and the energy is unbounded. This means that if one can create a tachyon and send it off in a given direction, its antiparticle must be created out along that direction somewhere with just the right direction and energy to meet the original tachyon at their duly appointed place of annihilation. To see that this can cause trouble, consider the creation of a $10^{20} \mathrm{eV}$ tachyon of electronic charge and mass sent off in the direction of the moon. As we have seen, its range to annihilation is $11 \mathrm{~km}$. If its anti-particle is created on the moon in order to have sufficient range to reach the annihilation point, it would need an energy at point of origin $\approx 10^{24} \mathrm{eV}$. This situation quickly gets out of hand if, instead of the moon, we aim our tachyon at the nearest star.

The problem is clear; we may not employ "particles coming in from infinity" when applying the reinterpretation principle here. The incoming antiparticles must have a real source at a finite distance and the closer the better. When an experimenter creates a tachyon moving in a given direction, a source of anti-tachyons must be somewhere out along that direction. The only escape 
from this conclusion is to assert that if there is no anti-tachyon source in that direction, the experimenter will be forbidden, in some as yet unknown manner, to send his tachyon in that direction.

These considerations, of course, do not establish the non-existence of tachyons. They do indicate, however, that if they exist in a meaningful way the physics of such particles is going to appear very strange.

Acknowledgments

The author would like to thank many of his colleagues in the Theoretical Studies Branch, Goddard Space Flight Center for many discussions concerning this work. Special thanks are due Dr. R. J. Drachman for discussions concerning the manuscript as well as the general problem. 


\section{APPENDIX}

We begin with the wave equation for the potential

$$
\begin{aligned}
& \left(\partial_{x}^{2}+\partial_{y}^{2}+\partial_{z}^{2}-\partial_{t}^{2}\right) \Phi=-4 \pi \rho(x, y, z, t) \\
& \left(\partial_{x}^{2}+\partial_{y}^{2}+\partial_{z}^{2}-\partial_{t}^{2}\right) \vec{A}=-4 \pi \vec{j}(x, y, z, t)
\end{aligned}
$$

If $\rho$ represents a fixed charge distribution whose motion is a simple translation with velocity $\mathrm{v}$ in the $\mathrm{z}$ direction then

$$
\begin{gathered}
\rho=\rho(\mathbf{x}, \mathbf{y}, \mathbf{z}-\mathrm{v} \mathrm{t}), \Phi=\Phi(\mathbf{x}, \mathbf{y}, \mathbf{z}-\mathrm{v} \mathrm{t}), \vec{j}_{z}=\vec{v} \rho \\
\overrightarrow{\mathrm{A}}=\mathrm{A}_{\mathbf{z}} \hat{\mathrm{e}}_{\mathbf{z}}=\hat{\mathrm{e}}_{\mathbf{z}} \mathrm{v} \Phi
\end{gathered}
$$

Since from $(A-3)$ we have $\partial_{t}=-v \partial_{z}(A-1)$ becomes

$$
\left(\partial_{\mathrm{x}}^{2}+\partial_{\mathrm{y}}^{2}-\left(\mathrm{v}^{2}-1\right) \partial_{\mathrm{z}}^{2}\right) \Phi=-4 \pi \rho(\mathrm{x}, \mathrm{y}, \mathrm{z})
$$

where we have now transformed to a co-moving coordinate system $\mathrm{z}_{\mathrm{new}}=$ $\mathrm{z}_{\text {old }}$ - vt. (A-4) may be readily solved by Fourier transforming in $x, y$, and $\mathrm{z}$ to obtain

$$
\widetilde{\Phi}\left(\mathrm{k}_{\mathrm{x}}, \mathrm{k}_{\mathrm{y}}, \mathrm{k}_{\mathrm{z}}\right)=-\frac{4 \pi \tilde{\rho}\left(\mathrm{k}_{\mathrm{x}}, \mathrm{k}_{\mathrm{y}}, \mathrm{k}_{\mathrm{z}}\right)}{\mathrm{k}_{\mathrm{x}}^{2}+\mathrm{k}_{\mathrm{y}}^{2}-\mathrm{k}_{\mathrm{z}}^{2} / \gamma_{\mathrm{s}}^{2}}
$$


where

$$
\gamma_{s}=\left(v^{2}-1\right)^{-1 / 2}
$$

For an observer at a point $x, y$ from the charge's trajectory the frequency dependence of the potential will be given by $\widetilde{\Phi}\left(\mathrm{x}, \mathrm{y}, \mathrm{k}_{\mathrm{z}}\right)$ with $\omega=\mathrm{vk}_{\mathrm{z}}$. We have

$\tilde{\Phi}\left(\mathrm{x}, \mathrm{y}, \mathrm{k}_{\mathrm{z}}\right)=\frac{-1}{\pi} \int_{-\infty}^{\infty} \int \frac{\tilde{\rho}\left(\mathrm{k}_{\mathrm{x}}, \mathrm{k}_{\mathrm{y}}, \mathrm{k}_{\mathrm{z}}\right) \exp \left(\mathrm{i} \mathrm{k}_{\mathrm{x}} \mathrm{x}+\mathrm{i} \mathrm{k}_{\mathrm{y}} \mathrm{y}\right)}{\mathrm{k}_{\mathrm{x}}^{2}+\mathrm{k}_{\mathrm{y}}^{2}-\mathrm{k}_{\mathrm{z}}^{2} / \gamma_{\mathrm{s}}^{2}} \mathrm{~d} \mathrm{k}_{\mathrm{x}} \mathrm{d} \mathrm{k}_{\mathrm{y}}$.

If we now assume cylindrical symmetry for the charge distribution i.e.,

$$
\tilde{\rho}\left(\mathrm{k}_{\mathrm{x}}, \mathrm{k}_{\mathrm{y}}, \mathrm{k}_{\mathrm{z}}\right)=\tilde{\rho}\left(\mathrm{k}_{1}, \mathrm{k}_{\mathrm{z}}\right)
$$

where

$$
\mathrm{k}_{1}^{2}=\mathrm{k}_{\mathrm{x}}^{2}+\mathrm{k}_{\mathrm{y}}^{2}
$$

(A-6) becomes

$$
\begin{aligned}
\tilde{\Phi}\left(r, k_{z}\right) & =\frac{-1}{\pi} \int_{0}^{\infty} k_{1} d k_{1} \int_{0}^{2 \pi} \frac{d \phi \tilde{\rho}\left(k_{\perp}, k_{z}\right) \exp \left(i k_{\perp} r \cos \phi\right)}{k_{\perp}^{2}-k_{z}^{2} / \gamma_{s}^{2}} \\
& =-2 \int_{0}^{\infty} \frac{J_{0}\left(k_{\perp} r\right) \tilde{\rho}\left(k_{1}, k_{z}\right) k_{\perp} d k_{\perp}}{k_{\perp}^{2}-k_{z}^{2} / \gamma_{s}^{2}}
\end{aligned}
$$

where $J_{0}(z)$ is the Bessel function of zero order.

To evaluate the integral over $k_{1}$ we first express the Bessel function in terms of Hankel function as 


$$
J_{0}\left(k_{\perp} r\right)=\frac{1}{2}\left[H_{0}^{(1)}\left(k_{\perp} r\right)+H_{0}^{(2)}\left(k_{\perp} r\right)\right]
$$

Hankel functions are analytic in the plane cut from $-\infty$ to 0 along the negative real axis. In this cut plane they have the following symmetry and asymptotic properties;

$$
\left.\begin{array}{c}
\mathrm{H}_{0}^{(1)}\left(z \mathrm{e}^{\pi \mathrm{i}}\right)=-\mathrm{H}_{0}^{(2)}(\mathrm{z}) \\
\mathrm{H}_{0}^{(2)}\left(z \mathrm{e}^{-\pi \mathrm{i}}\right)=-\mathrm{H}_{0}^{(1)}(\mathrm{z}) \\
\mathrm{H}_{0}^{(1)}(\mathrm{z}) \sim(2 / \pi \mathrm{z})^{1 / 2} \operatorname{exp~i}\left(z-\frac{1}{4} \pi\right) \\
\mathrm{H}_{0}^{(2)}(\mathrm{z}) \sim(2 / \pi z)^{1 / 2} \exp -\mathrm{i}\left(z-\frac{1}{4} \pi\right)
\end{array}\right\} \mathrm{z} \rightarrow \infty
$$

From $(A-9)$ we see that we may extend the integral $(A-7)$ to the negative real axis as long as we stay above (below) the branch cut for the term containing $\mathrm{H}_{0}^{(1)}$ $\left(\mathrm{H}_{0}^{(2)}\right)$. It is readily shown that if

$$
\rho\left(|\mathbf{r}|>\mathbf{a}_{0}\right)=0
$$

then

$$
|\tilde{\rho}(\mathrm{k})| \cdot<\mathrm{K} \exp \left(|\mathrm{k}| \cdot \mathrm{a}_{0}\right) \text { as }|\mathrm{k}| \rightarrow \infty
$$

therefore for points outside the charge $\left(\mathrm{r}>\mathrm{a}_{0}\right)$ the term containing $\mathrm{H}_{0}^{(1)} \cdot\left(\mathrm{H}_{0}^{(2)}\right)$ vanishes exponentially on the upper (lower) infinite semicircle and the contours may be closed accordingly. The zeros of the denominator are moved off of the 
real axis by adding $i \in$ to the denominator, a prescription that guarantees outgoing rather than incoming waves. If we further assume that $\tilde{\rho}\left(\mathrm{k}_{1}, \mathrm{k}_{\mathrm{z}}\right)$ has no poles in the finite $k_{\perp}$ plane the integral $(A-7)$ may be evaluated by residues in a straightforward manner to obtain:

$$
\widetilde{\Phi}\left(r, k_{z}\right)=-\pi i H_{0}^{(1)}\left(k_{z} r / \gamma_{s}\right) \tilde{\rho}\left(k_{z} / \gamma_{s}, k_{z}\right)
$$

The field strengths are given by

$$
\begin{aligned}
& E_{z}=-\partial_{z} \Phi-\partial_{t} A_{z}=\left(v^{2}-1\right) \partial_{z} \Phi \\
& H_{\phi}=-\partial_{r} A_{z}
\end{aligned}
$$

which are Fourier transformed to become

$$
\begin{aligned}
& \widetilde{\mathrm{E}}_{z}=\left(v^{2}-1\right)\left(i k_{z}\right) \tilde{\Phi} \\
& \widetilde{\mathrm{H}}_{\phi}=-v \partial_{r} \tilde{\Phi}
\end{aligned}
$$

The radial component of Poyntings vector is given by

$$
S_{r}(r, z)=\frac{-1}{4 \pi} E_{z}(r, z) H_{\phi}(r, z)
$$

and the energy radiated per unit time is given by 


$$
\begin{aligned}
\frac{d w}{d t} & =2 \pi r \int_{-\infty}^{\infty} S_{r}(r, z) d z=-\frac{r}{2} \int_{-\infty}^{\infty} E_{z} H_{\phi} d z \\
& =-\frac{r}{2} \int_{-\infty}^{\infty} d z \int_{\infty}^{\infty} \frac{d k_{z} d k_{z}^{\prime}}{4 \pi^{2}} \tilde{E}_{z}\left(r, k_{z}\right) \tilde{H}_{\phi}\left(r, k_{z}^{\prime}\right) \operatorname{exp~} i\left(k_{z}+k_{z}^{\prime}\right) z \cdot(A-15) \\
& =-\frac{r}{4 \pi} \int_{-\infty}^{\infty} \tilde{E}_{z}\left(r, k_{z}\right) \tilde{H}_{\phi}\left(r,-k_{z}\right) \cdot d k_{z}
\end{aligned}
$$

Inserting $(A-13)$ in $(A-15)$ we obtain

$$
\begin{aligned}
\frac{d w}{d t} & =-\frac{r v\left(v^{2}-1\right)}{4 \pi} \int_{-\infty}^{\infty}\left(i k_{z}\right) \tilde{\Phi}\left(k_{z}\right) \partial_{r} \tilde{\Phi}\left(-k_{z}\right) d k_{z} \\
& =\frac{\pi r v\left(v^{2}-1\right)}{4 \gamma_{s}} \int_{-\infty}^{\infty}\left(-i k_{z}^{2}\right)\left|\tilde{\rho}\left(k_{z} / \gamma_{s}, k_{z}\right)\right|^{2} H_{0}^{(1)}\left(\frac{k_{z} r}{\gamma_{s}}\right) H_{1}^{(1)}\left(-\frac{k_{z} r}{\gamma_{s}}\right) d k_{z}
\end{aligned}
$$

If we now add the positive and negative values of $\mathrm{k}_{\mathrm{z}}$ together we obtain

$$
\frac{\mathrm{dw}}{\mathrm{dt}}=\frac{\pi \mathrm{rv}\left(\mathrm{v}^{2}-1\right)}{4 \gamma_{\mathrm{s}}} \int_{0}^{\infty}\left(-\mathrm{i} \mathrm{k}_{\mathrm{t}}^{2}\right)\left|\widetilde{\rho}\left(\mathrm{k}_{\mathrm{z}} / \gamma_{\mathrm{s}}, \mathrm{k}_{\mathrm{z}}\right)\right|^{2} \mathrm{~W}\left(\frac{\mathrm{k}_{\mathrm{z}} \mathrm{r}}{\gamma_{\mathrm{s}}}\right) \mathrm{d} \mathrm{k_{z }}
$$

where

$$
\begin{aligned}
W(z) & =H_{0}^{(1)}(z) H_{1}^{(1)}(-z)+H_{0}^{(1)}(-z) H_{1}^{(1)}(z) \\
& =H_{0}^{(1)}(z) H_{1}^{(2)}(z)-H_{0}^{(2)}(z) H_{1}^{(1)}(z) \\
& =4 i / \pi z
\end{aligned}
$$


Combining (A-18) with (A-17) gives

$$
\frac{\mathrm{d} w}{\mathrm{~d} t}=\mathrm{v}\left(\mathrm{v}^{2}-1\right) \int_{0}^{\infty}\left|\tilde{\rho}\left(\mathrm{k}_{\mathrm{z}} / \gamma_{\mathrm{s}}, \mathrm{k}_{\mathrm{z}}\right)\right|^{2} \mathrm{k}_{\mathrm{z}} \mathrm{d} \mathrm{k}_{\mathrm{z}}
$$




\section{REFERENCES}

1. S. Tanaka, Progr. Theor. Phys. (Kyoto) 24, 177 (1960).

2. O. M. P. Bilaniuk, V. K. Deshpande, and E. C. G. Sudarshan, Am. J. Phys. $\underline{30}, 718$ (1962).

3. G. Feinberg, Phys. Rev. 159, 1089 (1967).

4. M. E. Arons and E. C. G. Sudarshan, Phys. Rev. 173, 1622 (1968); J. Dhar and E. C. G. Sudarshan, Phys. Rev. 174, 1808 (1968).

5. A Peres, Lett. Nuovo Cimento 1, 837 (1969).

6. D. G. Boulware, Phys. Rev. D 1,2426 (1970).

7. R. Fox, C. G. Kuper, and S. G. Lipson, Proc. Roy. Soc. Lond. 316, 515 (1970); A. Bers, R. Fox, C. G. Kuper and S. G. Lipson, in Relativity and Gravitation, edited by C. G. Kuper and A. Peres (Gordon and Breach Science Publishers, Ltd. London, 1971), p. 41. Also see ref. 1 and H. Schmidt, Z. Physik 151, 365 (1958); 151, 408 (1958).

8. S. A. Bludman and M. A. Ruderman, Phys. Rev. D1, 3243 (1970).

9. R. G. Newton, Phys. Rev. 162, 1274 (1967); Science 167, 1569 (1970). For further references see J. S. Danburg, G. R. Kalbfleisch, S. R. Borenstein, R. C. Strand, V. VanderBurg, J. W. Chapman and J. Lys, Phys. Rev. D4, 53 (1971).

10. J. A. Parmentola and D. D. H. Yee, Phys. Rev. D4, 1912 (1971); A. F. Antippa and A. E. Everett, Phys. Rev. D4, 2198 (1971).

11. L. S. Schulman, Am. J. Phys. 39, 477 (1971). 
12. T. Alväger and M. N. Kreisler, Phys. Rev. $\underline{171}$, 1357 (1968).

13. M. B. Davis, M. N. Kreisler, and T. Alväger, Phys. Rev. $\underline{183,} 1132$ (1969).

14. A. S. Lapedes and K. C. Jacobs, Nature Physical Sci. $\underline{235}, 6$ (1972).

15. J. Weber, Phys. Rev. Lett. 25,180 (1970).

16. This has been pointed out by M. Glück, Nuovo Cimento $\underline{1 \mathrm{~A}}, 467$ (1971), however we cannot agree with his conclusion that charged tachyons would not emit Cherenkov radiation for his argument, if valid, would apply equally well in those cases where such radiation has been experimentally observed.

17. A Sommerfeld, Proc. Roy. Acad. Amsterdam (K. Akad. Wet. Amsterdam) 7, 346 (1904). A summary of this work can be found in A. Sommerfeld, Optics (Academic Press, New York, 1954) p. 328. It should be noted that in the latter work the author has the electric field within the shock cone pointing in the wrong direction; the field of a positive charge actually points towards the charge inside the shock cone. This apparent violation of Gauss's Law is clearly explained by G. M. Volkoff, Am. J. Phys. 31, 601 (1963).

18. I. Frank and Ig. Tamm, Compt. Rend. (Doklady) Acad. Sci. URSS 14, 109 (1937).

19. Ig. Tamm, J. Phys. (USSR) 1,439 (1939).

20. J. D. Jackson, Classical Electrodynamics (John Wiley and Sons, New York, 1962) p. 494.

21. P. A. M. Dirac, Proc. Roy. Soc. $\underline{167 A}, 148$ (1938); Ann. Inst. Poincare $\underline{9}, 13$ (1938). 
22. For a comprehensive review of radiation reaction theories for particles with $\mathrm{v}<1$ see T. Erber, Fortschr. Physik 9 , 343 (1961).

23. G. A. Schott, Electromagnetic Radiation (Cambridge Univ. Press, London, 1912).

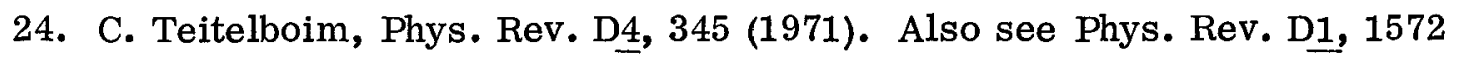
(1970); $\underline{3}, 297$ (1971).

25. Gravitational radiation from an extended tachyon was considered for the transcendent $(\mathrm{V}=\infty)$ case only by L. S. Schulman, Nuovo Climento $2 \underline{\mathrm{B}}, 38$ (1971), however, he considered the world line to be straight and of infinite length, an assumption that is inconsistent with the shape of the world line derived here. 